\title{
Amplification for adults with hearing difficulty, speech in noise problems - and normal thresholds
}

Keywords: amplification, adults, hearing difficulty, speech, normal thresholds, signal-to-noise ratios

\section{Introduction}

Most physicians and audiologists know that hearing difficulty (HD) and/or the inability to understand speech-in-noise (SIN) are chief complaints for some 38 million people with sensorineural hearing loss (SNHL) in the United States. These are neither trivial nor rare complaints. Rather, they represent a nearly universal descriptor of the most typical auditory complaints of most people with SNHL. However, few professionals are likely aware that these same "suprathreshold" complaints are voiced by an additional 26 million people who have hearing thresholds within normal limits (WNL), when tested with a standard audiologic test battery in quiet. People who consult physicians and audiologists complaining of HD or SIN problems in the presence of thresholds WNL often present a dilemma for hearing professionals. Specifically, they have normal hearing on standard tests, but still complain of SIN and HD problems. Of course, the given scenario begs the question, "What is the appropriate resolution for these problems?"

Persons with supra-threshold complaints and normal thresholds are often counseled about taking advantage of "happy talk" strategies. That is, they are generally told something along the lines of "The good news is you have normal hearing..." and they are instructed to use better lighting, and perhaps to focus on visual cues and/or visual redundancy, and to reduce the distance between the talker and the listener and more. However, although these strategies may offer some benefit, they may also frustrate patients further and may not be accepted as being particularly helpful or realistic solutions to their problems. Of note, when adults report they only have difficulty in challenging acoustic situations or difficulty understanding SIN, they are usually reporting accurate and sophisticated observations. Further, because they are adults, it is likely they have already tried many of these same apparent and obvious solutions without substantial success and have only come to see hearing care professionals after having exhausted these same solutions.

Unfortunately, many supra-threshold complaints are overlooked, or are not treated seriously, and may be explained as due to "normal aging" or ignored. Thus, many of these individuals "slip through the cracks" or fail to receive early intervention for their HDs and/or SIN problems. Delays in diagnosis and treatment often perpetuate these patients' problems and can affect the overall quality of life (QoL) for them and their families. Physicians and audiologists can and should do more for people with supra-threshold auditory complaints.

\section{Best practices}

Many physicians and audiologists practice with an eye toward detecting otologic diseases which may require medical or surgical interventions, and as anticipated, the vast majority of these same problems (i.e., those in need of medical or surgical attention) are identified by the standard audiologic battery (i.e., air- and boneconduction pure tones and speech audiometry in quiet). Unfortunately,
Volume II Issue I - 2019

\author{
Douglas L Beck,' Jeffrey L Danhauer² \\ 'Oticon inc, Executive Director of Academic Sciences, USA \\ ${ }^{2}$ Emeritus Professor of Audiology, University of California Santa \\ Barbara, USA
}

Correspondence: Douglas L Beck, Au.D, Executive Director of Academic Sciences, Oticon Inc., 580 Howard Ave, Somerset, N] 08873, USA, Tel 732-673-4048,Email dbec@oticon.com

Received: January 31, 2019 | Published: February 18, 2019

this same battery often fails to detect listening difficulties and may not accurately reflect how patients function in their daily lives. Therefore, many patients are likely not identified or informed about available tools designed to improve signal-to-noise ratios (SNRs) which could alleviate or reduce their HD or SIN problems. Indeed, hearing loss is not the primary concern for people with normal thresholds who complain of hearing difficulty or SIN problems, the primary issue is measuring, documenting and improving the signal-to-noise ratio (SNR) required to understand SIN. Importantly, improving the SNR is "always helpful to a listener." Had these same individuals been tested and managed according to the American Academy of Audiology (AAA) or the American Speech Language Hearing Association (ASHA) Best Practice (BP) guidelines, ${ }^{2}$ it is likely their HD and/or SIN problems would be discovered, documented, and scheduled for appropriate treatments in a timely manner.

Patients' complaints of HD and SIN problems in the absence of peripheral hearing loss are not new, and are in fact, quite common. The research literature reveals many studies have reported patients' complaints of HDs in the presence of normal thresholds in 10 to $50 \%$ of their subjects, especially in older persons (e.g., Saunders and Haggard, ${ }^{3}$; Gates et al., ${ }^{4}$ Chia et al., ${ }^{5}$ Hannula et al., ${ }^{6}$ Tremblay et al., ${ }^{7}$ Spankovich et al. ${ }^{8}$ )

Clearly, HDs and SIN problems are not limited to abnormal audiometric thresholds. According to Beck, Larsen, and Bush, ${ }^{9}$ people with hearing thresholds WNLs may have supra-threshold auditory deficits due to or associated with any of the following: central presbycusis, auditory disability with normal hearing, obscure auditory dysfunction (OAD), King-Kopetzky Syndrome, auditory dysacusis, auditory processing disorders (APDs), idiopathic discriminatory dysfunction, hidden hearing loss (HHL), cochlear synaptopathy (CS), tinnitus, neurocognitive disorders, dyslexia, attention deficit disorder (ADD), attention deficit hyperactivity disorder (ADHD), traumatic brain injury (TBI), spatial hearing disorders (SHDs), aging, dementia, cognitive decline, presbycusis (sensory, neural, synaptic, auditory fiber, and/or central causes), noise-induced hearing loss (NIHL), mild cognitive impairment (MCI), receptive aphasia, and Alzheimer's Disease among others.

Some physicians and audiologists may not be familiar with recent research and clinical experience that have identified a recently recognized auditory disorder, cochlear synaptopathy (CS) or hidden hearing loss (HHL), that could be a cause of or contributor to HDs and 
SIN problems in people with otherwise normal hearing. ${ }^{10} \mathrm{CS} / \mathrm{HHL}$ involves problems with the ribbon fibers that synapse the cochlear hair cells to the auditory nerve fibers and this condition is receiving considerable research attention. ${ }^{10,11}$ Although there is presently no empirically validated test battery to diagnose CS/HHL, researchers are evaluating several measures which may soon prove useful in making such diagnoses. ${ }^{11-13}$

\section{How are HD and SIN problems discovered and documented?}

Traditional audiology-based and validated self-assessment tools (e.g., the Client-Oriented Scale of Improvement, COSI; the Hearing Handicap Inventory for Adults, HHIA; the Hearing Handicap Inventory of the Elderly, HHIE; the Personal Assessment of Communication Ability, PACA; and the Abbreviated Profile of Hearing Aid Benefit, APHAB) can be used to assess common hearing problems and difficulties that occur in daily life. ${ }^{14}$ These self-assessment tools query patients about the perceived quantity and quality of their hearing difficulties and/or SIN problems. These measures may also be helpful for identifying HD and SIN problems in patients with thresholds WNL.

\section{Speech-in-noise tests}

In addition to self-assessment measures, several objective SIN tests having high validity and test reliability are available for use with adults and can be administered in only a few minutes per test. Among the most popular SIN tests are the Bamford-Kowal-Bench Speech-inNoise test (BKB-SIN), the Words in Noise Test (WIN), the Hearing in Noise Test (HINT) the Quick-SIN and the Speech Perception in Noise (SPIN) test (for a review of many of these tests, see Wilson. ${ }^{15}$ Several protocols are used in SIN testing. Stimuli may be presented via fixed or adaptive protocols and may include natural sentences, spondees, or monosyllabic words. Multi-talker babble (four or more talkers) is a preferred noise competitor because its speech/linguistic composition is more representative of typical restaurant, cocktail party, train station, and other noisy llistening situations rather than, speech spectrum, white, and other artificial noises that lack linguistic information and are generally easier to ignore. ${ }^{16}$ Regardless, of the particular test selected, we recommend local normative values should be determined.

To approximate SIN problems in the real world, SIN measures are acquired in a calibrated sound field using speakers (not headphones, not insert ear phones). The Signal-to-Noise Ratio required to achieve a $50 \%$ correct response rate is referred to as the "SNR-50." Based on clinical observations and reports based on the QuickSin, adults with thresholds WNL should achieve an SNR-50 of 0,1 , or $2 \mathrm{~dB}$. People with mild-moderate sensorineural hearing loss typically have an SNR-50 of 6, 7 or $8 \mathrm{~dB}$. However, some patients with thresholds WNL report significant problems understanding speech, and these people should be evaluated with SIN tests to determine their SNR50 , For example, if a patient has audiometric thresholds WNL, one might expect an SNR-50 of about 1 or $2 \mathrm{~dB}$; however, if that same patient presents with an SNR-50 of 8 or $9 \mathrm{~dB}$, then they clearly have significant difficulty understanding SIN. The goal for the clinician (after ruling out medical and/or surgical conditions) is to improve the SNR-50 as much as possible. For example, given normal thresholds and an SNR-50 of $8 \mathrm{~dB}$, this would indicate the need for tools which can improve the SNR-50 by approximately $6 \mathrm{~dB}$. Of note, simply providing amplification may not be enough as many amplification technologies amplify the background noise along with the primary speech signal, rendering a louder sound with a negligible change in the SNR. Therefore, anticipated improvements in the SNR-50 must be verified and validated using the same test conditions as used in the unaided measures.

Wilson $^{17}$ reported the SIN ability of 3024 veterans from 20 to 80 years of age with pure-tone averages between 15-70 dB HL. Because neither speech in quiet nor pure-tones predicted listeners' SIN ability, Wilson recommended including SIN testing in every audiologic diagnostic assessment. However, although AAA and ASHA Best Practice (BP) statements recommend SIN testing as part of comprehensive adult audiologic evaluations, fewer than $15 \%$ of audiologists perform it routinely. ${ }^{2,18}$ Thus, audiologists should reconsider using SIN tests, especially with patients complaining of HD and/or SIN problems in the presence of normal thresholds.

\section{Hearing aids can improve HDs and SIN problems}

Fitting hearing aids to patients (even those with thresholds WNL) based on their perceived HDs is not a new concept. ${ }^{19}$ Physicians and audiologists need to know that many people with HDs and SIN problems, including those with near-normal thresholds, may benefit from contemporary hearing aids fitted to BP standards. Most patients with normal or near-normal thresholds, who complain of HD and/or SIN problems and try hearing aids, elect to purchase them at the end of their trial periods. ${ }^{20}$

Beck \& Le Goff ${ }^{21}$ found statistically significant improvements in word recognition scores and in SNRs for 25 older adults (mean age $=73$ years) who wore sophisticated hearing aids with directional technology, narrow-band beam-forming directionality, and multispeaker-access-technology (MSAT) when tested in two different types of noise originating from three different locations. They reported that statistically significant improvements were obtained with MSAT, which improved SIN scores and SNRs (6.3 dB on average).

Roup, Post \& Lewis ${ }^{22}$ described results for 20 normal-hearing adults (19-27 years of age) with no self-reported HDs and 19 normal-hearing adults (18-58 years of age) with self-reported HDs. All were fitted binaurally with mild-gain, receiver-in-the-canal (RIC) hearing aids for a four-week trial with directional and noise reduction algorithms engaged. SIN measures were acquired pre-and-post hearing aid fitting and most reported that the hearing aids helped in quiet $(67 \%)$ and in noise $(71 \%)$.

Ohlenforst, Wendt \& Colleagues $^{23}$ demonstrated that noise reduction protocols in contemporary hearing aids can reduce listening effort (as measured via peak pupil dilation, PPD), and noise reduction was found to increase word and sentence recognition in four-talker background noises, in various signal-to-noise ratios (SNRs). The authors emphasized the importance of attending to and measuring listening effort and speech in noise.

Beck, $\mathrm{Ng} \&$ Jensen $^{24}$ reported contemporary hearing aids have been shown to improve word recall in noise, improve SNRs, improve recall of words heard in noise, reduce listening effort, and facilitate higher satisfaction and more.

Similarly, mild-gain amplification may prove to be useful for some patients with CS/HHL based on anecdotal evidence and personal experience which indicates that some patients with CS/ HHL symptoms derive improvement for their complaints of HDs and tinnitus. Another potential treatment for CS/HHL uses auditory training to help alleviate symptoms in these patients. ${ }^{25}$ Although considerable research needs to be conducted regarding the diagnosis 
and treatments for CS/HHL, physicians and audiologists should know that mild-gain amplification is a viable treatment option for some of these individuals with HDs, but whose hearing thresholds are WNL.

\section{Remote microphone systems}

Hearing aids alone may not be enough to correct HDs and SIN problems for some patients. In that case, remote microphone (RM) systems can provide additional SNRs and other benefits. RM systems are radio devices that capture audio signals of interest and deliver them via radio frequency (RF) transmission to a radio receiver coupled to listeners' hearing aids or other receivers. RM systems are inexpensive and are available with most modern hearing aid systems and can substantially improve SNRs (in some cases by 10 to $20 \mathrm{~dB}$ ), reduce reverberation, and minimize deleterious effects of distance. ${ }^{26,27}$ Although historically, most RM systems transmitted audio signals via a frequency modulated (FM) radio signal, modern RM systems deliver audio signals from the microphone to radio receivers via digital RF transmission. These modern RMs can eliminate interference from other nearby RF systems, reduce noise and static which occasionally accompanies FM signals, offer a wider bandwidth, increase the sophistication of processing applied to transmitted signals, and produce excellent speech recognition in noise. ${ }^{28,29}$ Some RM users' SIN results approach those obtained by people with relatively normalhearing ability. ${ }^{30}$

RM systems can be coupled to many commercially available hearing aids and will significantly improve speech recognition in noise relative to the "no RM" condition at SNRs commonly encountered in real-world situations. ${ }^{29,31,32} \mathrm{RM}$ systems perform well for people with APD, dyslexia, autism spectrum disorder, and attention and other learning disorders. RM systems also have therapeutic benefits including improved cortical auditory-evoked potential, auditory brainstem response, frequency discrimination, binaural temporal resolution, frequency pattern recognition, and auditory working memory. RM systems have also been shown to improve attention, learning, and behavior, participation in class, self-esteem, and psychosocial development. RMs can enhance listening and provide substantial benefit to hearing-impaired persons, even in the most deleterious acoustically challenging listening environments with noise and reverberation. Adults with traumatic brain injuries (TBIs), particularly veterans, also benefit from improved SNRs provided by RMs. ${ }^{30}$

Physicians and audiologists are increasingly addressing the auditory needs of blast-exposed and TBI veterans. Often, these vets present with hearing thresholds WNL yet have HD, SIN, and/or tinnitus problems. In these situations, professionals should consider and measure patients' SIN ability (i.e., determine their SNR-50) listening and communication demands, auditory lifestyle, and other factors in order to diagnose and document their HD and SIN ability, which will facilitate the recommendation of tools which can produce successful outcomes $^{33}$. Hearing aids, particularly those coupled to RMs, can help these individuals, even in the presence of thresholds WNL., ${ }^{9,34}$ Many blast-exposed veterans complain of difficulty understanding SIN, rapid speech, and following instructions, often disproportionately to their peripheral hearing sensitivity. ${ }^{35} \mathrm{FM}$ and RM systems using Bluetooth are effective interventions for blast-exposed veterans with normal or near-normal hearing sensitivity, but HDs. These tools (and others which improve the SNR) should routinely be considered as a potential intervention approach along with mild-gain hearing aids for adults with APDs. ${ }^{36}$

People with HDs, including those with neurocognitive disorders and TBIs, often experience significant QoL benefits from sophisticated hearing aids coupled to FM and RM systems. These systems can reduce background noise, improve SNRs, enhance SIN outcomes, maintain and take advantage of realistic and naturally occurring binaural spatial cues that allow wearers to know where to focus their attention. These tools may soon be shown to serve a neuroprotective role against cognitive decline associated with hearing loss and some neurocognitive disorders. ${ }^{9,37}$

\section{Discussion}

Although clinicians generally do not advocate amplification for people with normal thresholds, many individuals with HDs and/or SIN problems perform better in social, professional, and recreational arenas when SNRs are improved via hearing aids, RMs, and other tools. In addition to some 38 million people in the USA with audiogram-based documented hearing loss, another 26 million adults may have HD or SIN problems, despite having hearing thresholds WNLs. Physicians need to know that once they have cleared patients medically, audiologists can documents their SIN and HDs by quantifying them using validated measures, improve their QoL via technology, assure that no damage is being done (using probe microphone real-ear measures), and allow patients a trial period to decide if the tools which provide improved SNRs are worth their time and money.

Sophisticated hearing aid technologies (i.e. MSAT) have been shown to improve the SNR by $6 \mathrm{~dB}$ or more. For many people, this represents a significant and useful improvement in SNR. Additionally, telecoils, digital remote microphones, BlueTooth and other wireless protocols can improve the SNR by an additional $10-15 \mathrm{~dB}$ or more. These technologies should be introduced and demonstrated to patients who complain of HD and/or SIN supra-threshold problems, despite normal or near-normal thresholds, to facilitate an improved QoL. who complain of HD and/or SIN supra-threshold problems despite normal or near-normal audiometric thresholds.

Person-centered care dictates that physicians and audiologists and patients engage in a decision-making process that encompasses the components of evidence-based practice founded on available research, expert opinion, and a demonstrated appreciation for patients' expressed experiences, needs, and concerns. ${ }^{38}$ Unfortunately, the typical medical-centric audiometric test battery may not be sensitive enough to pick up and validate many patients' supra-threshold complaints.

It is no longer appropriate for physicians and audiologists to tell patients their hearing is normal when supra-threshold auditory deficits are not explored or documented. ${ }^{39}$ Much like a cardiologybased stress test, SIN tests stress the auditory system and reflect performance in challenging situations. Traditional audiology tests only evaluate threshold conditions obtained under headphones in a sound-attenuating booth, which does not stress the auditory system or indicate how the person would perform under stress in the real world.

Almost everyone hears and listens better and is more relaxed in a quiet environment. People perceive and appreciate high-fidelity auditory sounds when acoustic information is received at favorable SNRs. Many individuals, even with normal thresholds, require or prefer enhanced SNRs and need improved access to auditory information to learn and participate in professional, social, recreational, and academic situations. Improving the brain's ability to listen and derive meaning from sound through technologies that improve the SNR can help remedy some persons' HD and SIN problems, reduce annoying effects of tinnitus, and ultimately enhance individuals' QoL. These technologies may soon prove to serve a neuroprotective role against 
cognitive decline associated with hearing loss and neurocognitive disorders. ${ }^{40-42}$ Therefore, physicians and audiologists should allow and encourage patients to be properly diagnosed according to AAA and ASHA Best Practice models, and when appropriate, allow and encourage these same technologies to be reviewed and demonstrated to patients with thresholds WNL, to improve their SIN ability, reduce their HDs and improve their QOL.

\section{Special acknowledgment}

This work is an abbreviated and updated version of a previously published article. Original citation: Beck DL, Danhauer JL, Abrams $\mathrm{HB}$, et al. Audiologic considerations for people with normal hearing sensitivity yet hearing difficulty and/or speech-in-noise problems. Hearing Review. 2018; 25(10) [Oct]:28-38.

\section{Conflicts of interest}

The author declares there is no conflict of interest.

\section{References}

1. Lentz JJ. In "Psychoacoustics: Perception of normal and Impaired Hearing With Audiology Applications." Chapter 8 "Clinical Implications. 2020:211.

2. Beck DL. Best practices in hearing aid dispensing: An interview with Michael Valente, PhD. Hearing Review. 2017;24(12):39-41.

3. Saunders GH, Haggard MP. The clinical assessment of obscure auditory dysfunction 1 . Auditory and psychological factors. Ear Hear. 1989;10(3):200-208.

4. Gates GA, Cooper JC Jr, Kannel WB, et al. Hearing in the elderly: the Framingham cohort, 1983-1985. Part I. Basic audiometric test results. Ear Hear. 1990;11(4):247-256.

5. Chia EM, Wang JJ, Rochtchina E, et al. Hearing impairment and healthrelated quality of life: the Blue Mountains Hearing Study. Ear and Hearing. 2007;28(2):187-95.

6. Hannula S, Bloigu R, Majamaa K, et al. Self-reported hearing problems among older adults: prevalence and comparison to measured hearing impairment. J Am Acad Audiol. 2011;22(8):550-559.

7. Tremblay KL, Pinto A, Fischer ME, et al. Self-Reported Hearing Difficulties Among Adults with Normal Audiograms: The Beaver Dam Offspring Study. Ear Hear. 2015;36(6):e290-299.

8. Spankovich C, Gonzalez VB, Su D. Self-reported hearing difficulty, tinnitus, and normal audiometric thresholds, The National Health and Nutrition Examination Survey 1999-2002. Hear Res. 2017;pii: S03785955(17)30418-5.

9. Beck DL, Larsen DR, Bush EJ. Speech in noise: hearing loss, neurocognitive disorders, aging, traumatic brain injury and more. Journal of Otolaryngology, ENT Research. 2018;10(4):199-205.

10. Kujawa, SG, Liberman MC. Hidden hearing Loss - The Problem and the Promise. Association for Research in Otolaryngology. 2016.

11. Johnson CE, Barbee CM, Danhauer JL, et al. Toward the Future of Early Intervention for Adult Hearing Loss. Semin Hear. 2018;39(2):221-226.

12. Liberman MC, Epstein MJ, Cleveland SS, et al. Toward a Differential Diagnosis of Hidden Hearing Loss in Humans. PLoS ONE. 2016;11(9): e0162726.

13. Barbee CM, James JA, Park JH, et al. Effectiveness of Auditory Measures for Detecting Hidden Hearing Loss and/or Cochlear Synaptopathy: A Systematic Review. Semin Hear. 2018;39(2):172-209.
14. Johnson JA, Cox RM, Alexander GC. Development of APHAB norms for WDRC hearing aids and comparisons with original norms. Ear \& Hearing. 2010;31(1):47-55.

15. Wilson RH, McArdle RA, Smith SL. An Evaluation of the BKB-SIN HINT and QuickSIN, and WIN Materials on Lsteners with Normal Hearing and Listeners with hearing Loss. J Speech Lang Hear Res. 2007;50(4):844-856.

16. Beck DL, Nilsson M. Speech-in-noise testing: A pragmatic addendum to hearing aid fittings. Hearing Review. 2013;20(5):24-26.

17. Wilson RH. Clinical experience with the Words-in-Noise Test on 3430 Veterans: Comparison with pure tone thresholds and word recognition in quiet. $J$ Am Acad Audiol. 2011;22(7):405-423.

18. Clark, JG., Huff, C. and Earl, B. (2017): Clinical Practice Report Card - Are we meeting Best Practice Standards for Adult Hearing Rehabilitation? Audiology Today Nov/Dec. 15-25.

19. Winchester RA. When Is a Hearing Aid Needed? Maico Audiological Library Series. 1975;12:36-39.

20. Bennett C. Otolaryngol Head and Neck Surg. 1989;100:154.

21. Beck DL, Le Goff N. Contemporary Hearing Aid Amplification: Issues and Outcomes in 2018. Journal of Otolaryngology ENT Research 2018;7(3):18.

22. Roup CM, Post E, Lewis J. Mild-Gain Hearing Aids as a Treatment for Adults with Self-Reported Hearing Difficulties. $J$ Am Acad Audiol. 2018;29(6):477-494.

23. Ohlenforst B. Wendt D. Kramer SE. et al. Impact of SNR, masker type and noise reduction processing on sentence recognition performance and listening effort as indicated by the pupil dilation response. Hearing Research. 2018;365:90-99.

24. Beck DL, Ng E, Jensen JJ. A scoping review 2019: OpenSound Navigator. Hearing Review. 2019;26(2):28-31.

25. Spehar B, Lichtenhan J. Surveying Patients with 'Hidden Hearing Loss The Hearing Journal. 2018;72(12):28-30.

26. Mroz M. FM systems for people with hearing loss. 2018

27. Beck DL, Tomasula SJ. FM Made Friendly. 2006.

28. Thibodeau L. Comparison of speech recognition with adaptive digital and FM remote microphone hearing assistance technology by listeners who use hearing aids. Am J Audiol. 2014;23(2):201-210.

29. Wolfe J, Morais M, Schafer E, et al. Evaluation of speech recognition of cochlear implant recipients using a personal digital adaptive radio frequency system. J Am Acad Audiol. 2013;24(8):714-724.

30. Thibodeau LM, DeConde Johnson C. Wireless Technology to Improve Communication in Noise. Seminars in Hear. 2014;35(03):157.

31. Wolfe J, Duke M, Schafer E, et al. Evaluation of performance with an adaptive digital remote microphone system and a digital remote microphone audio-streaming accessory system. Am $J$ Audiol. 2015;24(3):440-450.

32. Wolfe J. Evaluation of modern remote microphone technologies. GN ReSound White Paper. 2018.

33. Saunders GH, Frederick MT, Arnold ML, et al. A Randomized Controlled Trial to Evaluate Approaches to Auditory Rehabilitation for Blast-Exposed Veterans with Normal or Near-Normal Hearing Who report Hearing Problems in Difficult Listening Situations. J Am Acad Audiol. 2018;29(1):44-62.

34. Gallun FJ, Papesh MA, Lewis MS. Hearing complaints among veterans following traumatic brain injury. Brain Inj. 2017;31(9):1183-1187. 
35. Saunders GH, Frederick MT, Chisolm TH, et al. Use of a FrequencyModulated System for Veterans with Blast Exposure, Perceived Hearing Problems, and Normal Hearing Sensitivity. Seminars in Hearing. 2014;35(03):227-238.

36. Smart JL, Kelly AS, Searchfield GD, et al. Rehabilitation of adults with auditory processing disorder and normal peripheral hearing: Two case studies. Aust N Z J Audiol. 2007;29(1):53-59.

37. Rodemerk KS, Galster JA. The Benefit of Remote Microphones with Four Different Wireless Protocols. J Am Acad Audiol. 2015;26(8):724231.

38. Clark JG, English KM. Counseling-Infused Audiologic Care. 3rd Ed. Inkus Press: Cincinnati, OH. 2019.
39. Roup C. The Impact of Minimal to Mild Sensorineural Hearing Loss in Adults. Perspectives of the ASHA Special Interest Groups. 2016;6(1):2.

40. Beck DL, Danhauer JL, Abrams HB, et al. Audiologic considerations for people with normal hearing sensitivity yet hearing difficulty and/or speech-in-noise problems. Hearing Review. 2018;25(10):28-38.

41. Kujawa SG, Liberman MC. Adding insult to injury: cochlear nerve degeneration after "temporary" noise-induced hearing loss. J Neurosci. 29(45):14077-14085.

42. Thibodeau LM, Schaper L. Benefits of Digital Wireless Technology for Persons with Hearing Aids. Seminars in Hearing. 35(03):168-176. 